\title{
Annealing effect of surface-activated bonded diamond/Si interface
}

\section{Jianbo Liang, Yan Zhou, Satoshi Masuya, Filip Gucmann, Manikant Singh, James Pomeroy, Seongwoo Kim, Martin Kuball, Makoto Kasu, Naoteru Shigekawa}

\begin{tabular}{|c|c|}
\hline Citation & Diamond and Related Materials, 93; 187-192 \\
\hline Issue Date & 2019-03 \\
\hline Type & Journal Article \\
\hline Textversion & Author \\
\hline Rights & $\begin{array}{l}\text { C } 2019 \text { Elsevier B.V. This manuscript version is made available under the } \\
\text { CC-BY-NC-ND } 4.0 \text { License. https://creativecommons.org/licenses/by-nc-nd/4.0/. } \\
\text { The following article has been accepted by Diamond and Related Materials. This is } \\
\text { the accepted manuscript version. The article has been published in final form at } \\
\text { https://doi.org/10.1016/j.diamond.2019.02.015. }\end{array}$ \\
\hline Highlights & $\begin{array}{l}\text { - Diamond/Si bonding interface could withstand a load of high temperature as high } \\
\text { as } 800^{\circ} \mathrm{C} \text {. } \\
\text { - The amorphous layer observed at the bonding interface decreased with annealing } \\
\text { temperature. } \\
\text { - The residual stress released in the diamond of the bonding interface decreased with } \\
\text { annealing temperature. } \\
\text { - The residual stress formed in the bonding interface annealed at } 1000^{\circ} \mathrm{C} \text { is much } \\
\text { smaller than that of diamond grown on Si. }\end{array}$ \\
\hline DOI & $10.1016 /$ j.diamond.2019.02.015 \\
\hline
\end{tabular}

Self-Archiving by Author(s)

Placed on: Osaka City University Repository 


\section{Annealing effect of surface-activated bonded diamond/Si interface}

Jianbo Liang ${ }^{\mathrm{a}}$, Yan Zhou ${ }^{\mathrm{b}, 1}$, Satoshi Masuya ${ }^{\mathrm{c}}$, Filip Gucmann ${ }^{\mathrm{b}}$, Manikant Singh ${ }^{\mathrm{b}}$, James Pomeroy ${ }^{\mathrm{b}}$, Seongwoo Kim ${ }^{\mathrm{d}}$, Martin Kuball ${ }^{\mathrm{b}}$, Makoto Kasu ${ }^{\mathrm{c}}$, and Naoteru Shigekawa ${ }^{\mathrm{a}}$

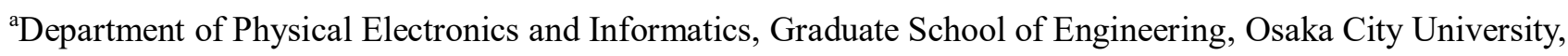
Sugimoto 3-3-138 Sumiyoshi-ku, Osaka 558-8585, Japan

${ }^{\mathrm{b}}$ Center for Device Thermography and Reliability (CDTR), University of Bristol, Bristol BS8 1TL, United Kingdom

${ }^{c}$ Department of Electrical and Electronic Engineering, Saga University, Honjo-machi 1, Saga 840-8502, Japan

${ }^{\mathrm{d}}$ Adamant Namiki Precision Jewel Co., Ltd., Shinden 3-8-22 Adachi-ku, Tokyo 123-8511, Japan

*Corresponding author E-mail address: liang@elec.eng.osaka-cu.ac.jp (J. Liang).

${ }^{1}$ Co-first authors.

Keywords: Direct bonding of diamond and Si, Bonding interface, Residual stress, Surface activated bonding, Interfacial structure.

From transmission electron microscope (TEM) observation, a $25 \mathrm{~nm}$ thick amorphous layer was confirmed at the diamond/Si bonding interface without annealing, the amorphous layer thickness decreased with the annealing temperature. No cracking even in nanometer scale occurred even after high-temperature annealing at $800{ }^{\circ} \mathrm{C}$. From in-situ micro-Raman monitoring, the compressed stress was observed in the $\mathrm{Si}$ of the bonding interface without annealing with respect to the bonding $\mathrm{Si}$ substrate. A tensile stress relaxation was observed in the diamond of the bonding interface with respect to the polished diamond. These results were attributed to the large load applied to the diamond and $\mathrm{Si}$ substrates during bonding process. After annealing at $400{ }^{\circ} \mathrm{C}$, the residual stress in the Si of the bonding 
interface decreased, while the residual stress in the diamond of the bonding interface increased, which should be due to the residual stress caused by the applied load was released by annealing process. The residual stress in the Si and diamond of the bonding interface increased and decreased, respectively, as the annealing temperature increased from 400 to $1000{ }^{\circ} \mathrm{C}$. The main reason was due to the structure change of the amorphous layer and the tensile stress relaxation in the diamond of the bonding interface.

\section{Introduction}

Diamond is expected to be the best potential candidate as the next generation semiconductor material for high power and high frequency electronic devices, due to its superior material properties such as high carrier mobility [1], high saturation velocity, high chemical inertness, the highest electrical breakdown field strength among semiconductor materials [2], and the highest thermal conductivity among materials. Therefore, diamond is also the most promising material as a superior heat spreading substrate for power devices. To date, diamond devices with high-current and low-loss capability [3-5], thermal stability [6-7], and fast switching with low switching losses [8] have been reported.

Large single crystal diamond (SCD) with high quality is necessary for fabricating diamond devices. However, high pressure high temperature (HPHT) synthesized SCD is limited to a surface area of about $1 \mathrm{~cm}^{2}$. Moreover, the cost of SCD is extremely expensive in comparison with that of Si. Recent years, the growth of SCD by microwave plasma chemical vapor deposition (CVD) on a foreign substrate has attracted considerable attention [9-13]. Several attempts to grow large SCD on SiC or Ir substrates by CVD have been conducted [14-18]. However, diamond thin films grown on these substrates contained many grain boundaries and appeared like poly-crystal diamond. Current semiconductor production line requires a minimum size of SCD in 2 inches for fabricating diamond devices. The lack of large SCD has prevented the development of diamond device technology and industrial applications.

The direct bonding of diamond and $\mathrm{Si}$ would be a very effective approach for overcoming the 
problems described above, which could effectively combine diamond device and Si Large-Scale Integration (LSI) with various functions on the same substrate and fabricate diamond device using Si LSI process facilities. A large numbers of small size diamonds bonded to a large diameter Si substrate could be used as a large diameter diamond substrate. We previously reported that the direct bonding of diamond and Si by surface activated bonding (SAB) at room temperature [19]. We demonstrated diamond/Si bonding interface with a full contact area of $4 \times 4 \mathrm{~mm}^{2}$ and good thermal stability at temperature of $1000{ }^{\circ} \mathrm{C}$ for $12 \mathrm{~h}$ [20]. Furthermore, we also demonstrated the fabrication of diamond field-effect transistor on the diamond bonded to Si. Although, the lattice constant (a) and thermal expansion coefficients $(\alpha)$ of diamond and $\mathrm{Si}$ are very different $\left(\mathrm{a}=3.57 \AA\right.$ and $\alpha=1.1 \times 10^{-6} / \mathrm{K}$ for diamond and $\mathrm{a}=5.43 \AA$ and $\alpha=3.9 \times 10^{-6} / \mathrm{K}$ for $\mathrm{Si}$ ), we achieved the direct bonding of diamond and $\mathrm{Si}$, which was attributed to the amorphous layer formed at the bonding interface by Ar fast atom beam irradiation during bonding process. It has been reported that the amorphous layers of the $\mathrm{Si} / \mathrm{Si}$ and $\mathrm{Si} / \mathrm{SiC}$ bonding interfaces disappeared after annealing at 600 and $1000{ }^{\circ} \mathrm{C}$, respectively, due to the recrystallization of the amorphous layer $[21,22]$. After the disappearance of the amorphous layer, a large thermal residual stress caused by the difference in thermal expansion coefficients between diamond and $\mathrm{Si}$ should be generated in the diamond/Si bonding interface fabricated by SAB. Moreover, the behavior of the bonding interface structures with annealing temperature is still unclear. These are very important to understand the mechanism of thermal stability of the diamond/Si bonding interface.

In this work, we investigate the structural properties of the diamond/Si bonding interface by transmission electron microscopy (TEM) and the residual stress in the diamond/Si bonding interface by confocal micro-Raman spectroscopy. In situ observation of microscopic structural changes in a TEM of the bonding interface at various annealing temperatures is carried out within a filament type heating TEM holder. The effect of thermal annealing process on the residual stress in the diamond/Si bonding interface is also investigated. The magnitude of the residual stress in the bonding interface is evaluated from the measured frequency shift with respect to the stress-free Raman peak position. 


\section{Experimental}

HPHT synthetic Ib type (100) single-crystal diamond substrates and Si (100) substrates were used for our bonding experiments. Diamond and Si substrates were purchased from Element Six Ltd. and MMT INC., respectively. The thickness and the size of the diamond and Si substrate is $550 \mu \mathrm{m}$ and 4 $\mathrm{mm} \times 4 \mathrm{~mm}$ and $525 \mu \mathrm{m}$ and $22 \mathrm{~mm} \times 25 \mathrm{~mm}$, respectively. The surface roughness of the diamond surface was measured using an atomic force microscope (AFM). As shown in Fig. 1, the averaged roughness $(\mathrm{Ra})$ of the diamond surface without polishing was determined to be $1.77 \mathrm{~nm}$, which is difficult to bond with other materials. The $\mathrm{Ra}$ value of the bonding material below $1 \mathrm{~nm}$ is crucial to achieve a successful bonding according to our dissimilar materials bonding experiences up to now. The diamond surface was polished by chemical-mechanical planarization (CMP). The Ra of the polished diamond surface was measured to be $0.32 \mathrm{~nm}$. Si and the polished diamond substrates were cleaned with acetone and ethanol in an ultrasonic bath for $300 \mathrm{~s}$, dried under $\mathrm{N}_{2}$, and then set in the chamber of SAB facilities. The background vacuum pressure of the chamber was kept as $5.0 \times 10^{-7} \mathrm{~Pa}$. The surfaces of diamond and Si substrates were activated by the Ar fast atom beams irradiation with a power of $1.5 \mathrm{kV}$ and $1.5 \mathrm{~mA}$. After the surface activation, diamond and Si were bonded to each other at room temperature by applying a pressure of $8 \mathrm{GPa}$, so that diamond/Si bonding interfaces were fabricated by SAB [23-25].

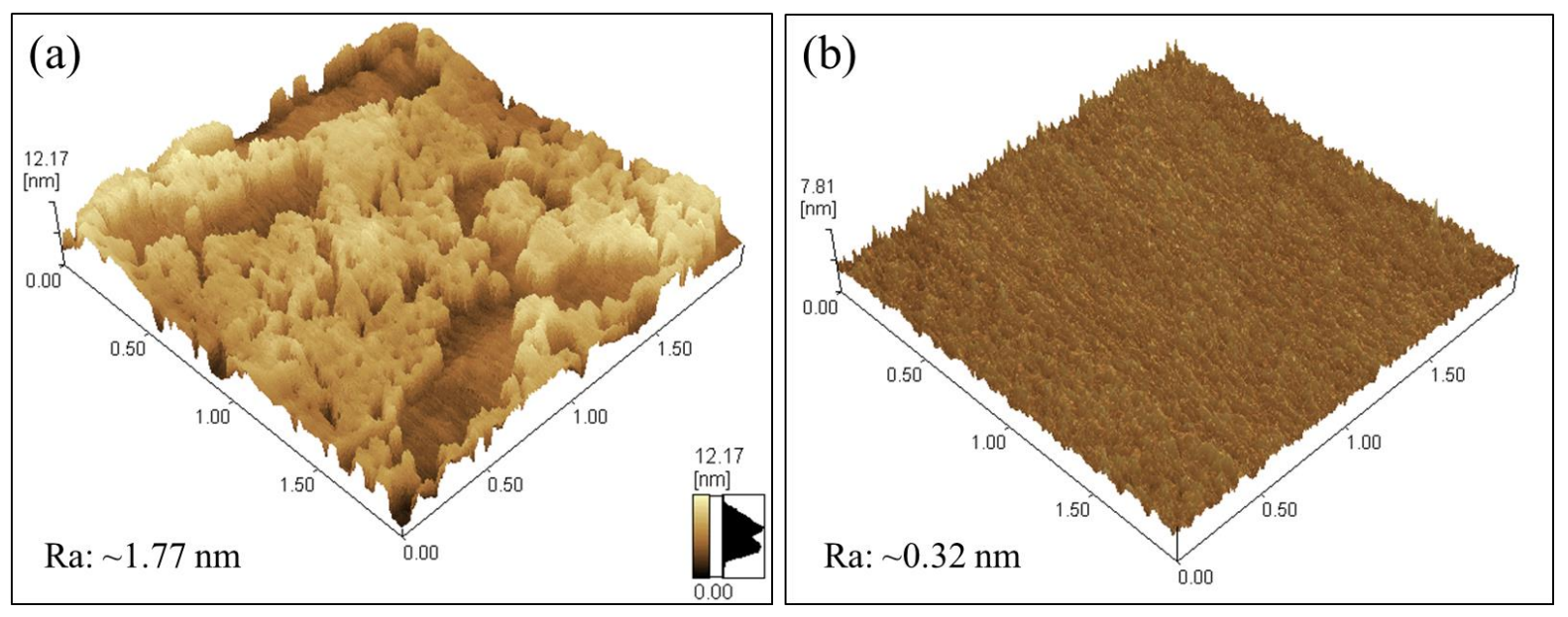

Fig. 1. AFM images of the diamond surface (a) without and (b) with chemical-mechanical planarization. 
The residual stress in the diamond/Si bonding interface was systematically investigated by microRaman spectroscopy. The effect of the annealing process on the residual stress of the bonding interface were investigated by in situ micro-Raman monitoring via a high-temperature heating stage (Linkam TS-1500) with a temperature stability of $\pm 1^{\circ} \mathrm{C}$. Diamond/Si bonding interfaces were annealed at 400 , 800 , and $1000{ }^{\circ} \mathrm{C}$ for $300 \mathrm{~s}$ in $\mathrm{N}_{2}$ gas atmosphere. Raman measurements were taken in backscattering geometry using a $488 \mathrm{~nm}$ Ar laser as excitation source, with an NA $0.650 \mathrm{x}$ objective to focus the laser and collect the Raman scattered light. Laser spot size on the sample was $0.5 \mu \mathrm{m}$. An area of $30 \times 30$ $\mu \mathrm{m}^{2}$ was scanned with $2 \mu \mathrm{m}$ steps to gain an average over the material properties to improve accuracy. The Si Raman line was used to calibration the Raman measurement. The Raman shift resolution was better than $0.1 \mathrm{~cm}^{-1}$. The diamond and Si peak position was determined using the curve-fitting function integrated in the operation software by performing a Lorentz fitting to the peaks. The interfacial structure of the bonding interface with annealing at different temperatures were investigated by in-situ TEM (JEM-2200FS) at room temperature under a high vacuum $\left(\sim 1 \times 10^{-5} \mathrm{~Pa}\right)$.

\section{Results and discussion}

Figs 2(a), 2(b), 2(c), and 2(d) show the low magnification cross-sectional TEM images of the diamond/Si bonding interface without and with annealing at 400,600 , and $800{ }^{\circ} \mathrm{C}$, respectively. A straight line separating from the surface of Si by approximately $300 \mathrm{~nm}$ could be clearly recognized in the figures, which corresponds to the bonding interface between diamond and Si. Note that the thin layer observed near the bonding interface was due to the formation of scalariform diamond thin film during TEM sample fabrication process. No interfacial voids or fracture defects even in a nanometer scale were observed at the bonding interface with annealing at different temperatures. Although the bonding interface was annealed at temperature as high as $800^{\circ} \mathrm{C}$, no any changes were observed at the bonding interface. 

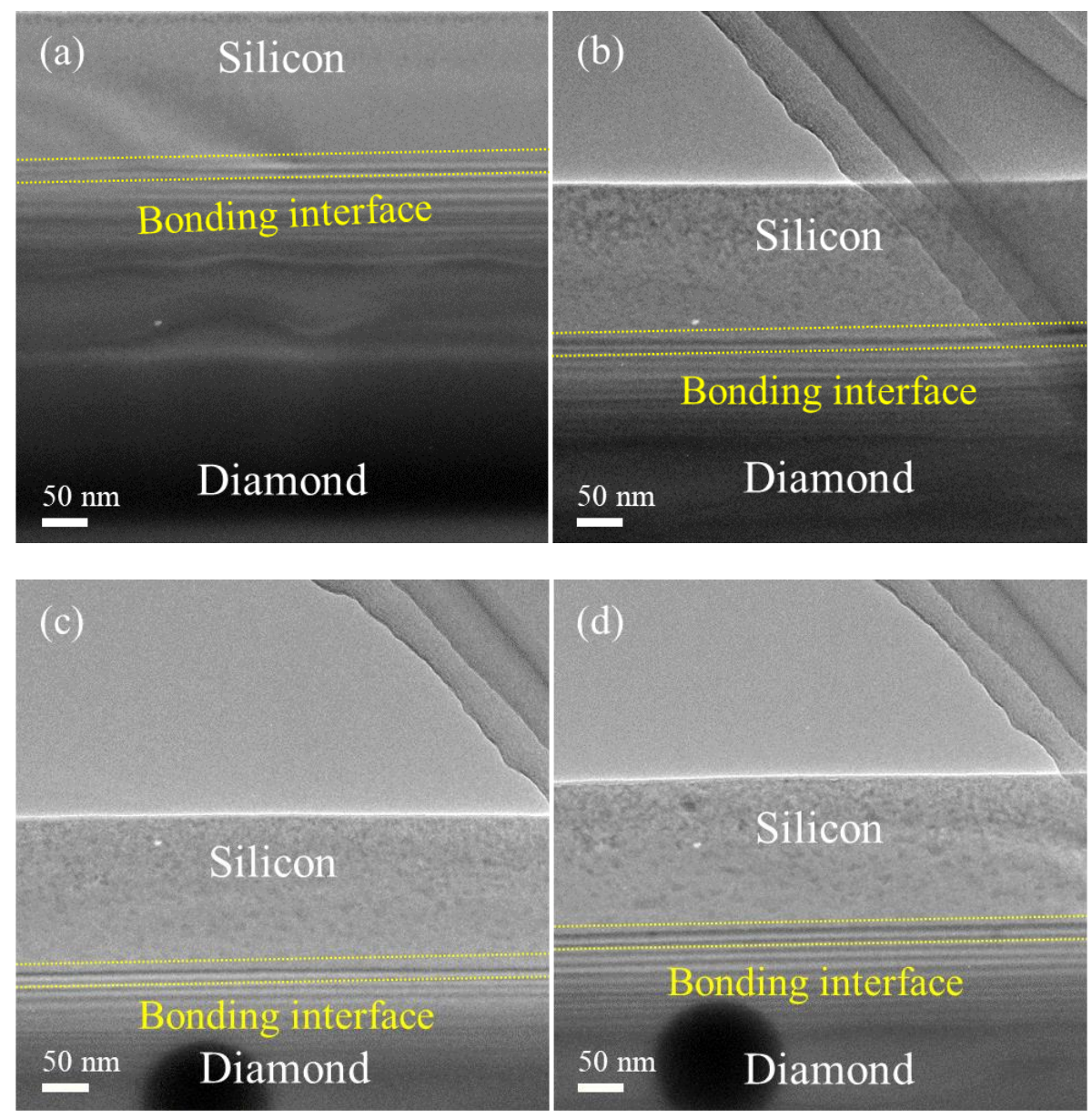

Fig. 2. low magnification cross-sectional TEM images of the diamond/Si bonding interface (a) without and with annealing at (b) 400, (c) 600 , and (d) $800{ }^{\circ} \mathrm{C}$.

The high magnification cross-sectional TEM images of the diamond/Si bonding interface without and with annealing at 400, 600, and $800^{\circ} \mathrm{C}$ are shown in Figs 3(a), 3(b), 3(c), and 3(d), respectively. As shown in Fig. 3(a), an amorphous layer with a thickness of about $25 \mathrm{~nm}$ was observed at the bonding interface, which is attributed to the surface damage caused by Ar beam irradiation during bonding process. The amorphous layer thickness was decreased from $25 \mathrm{~nm}$ to $17.5 \mathrm{~nm}$ by annealing at $400{ }^{\circ} \mathrm{C}$. When the annealing temperature increased to $600{ }^{\circ} \mathrm{C}$, the amorphous layer thickness was decreased to $6.7 \mathrm{~nm}$. Furthermore, the thickness was decreased further to $5.6 \mathrm{~nm}$ by annealing at $800{ }^{\circ} \mathrm{C}$. These results indicate that the amorphous layer thickness highly depended on the annealing temperature, which decreased with the annealing temperature. More importantly, there are no structural defects such as cracks in the nanometer scale observed at the interface with annealing at various temperatures. 

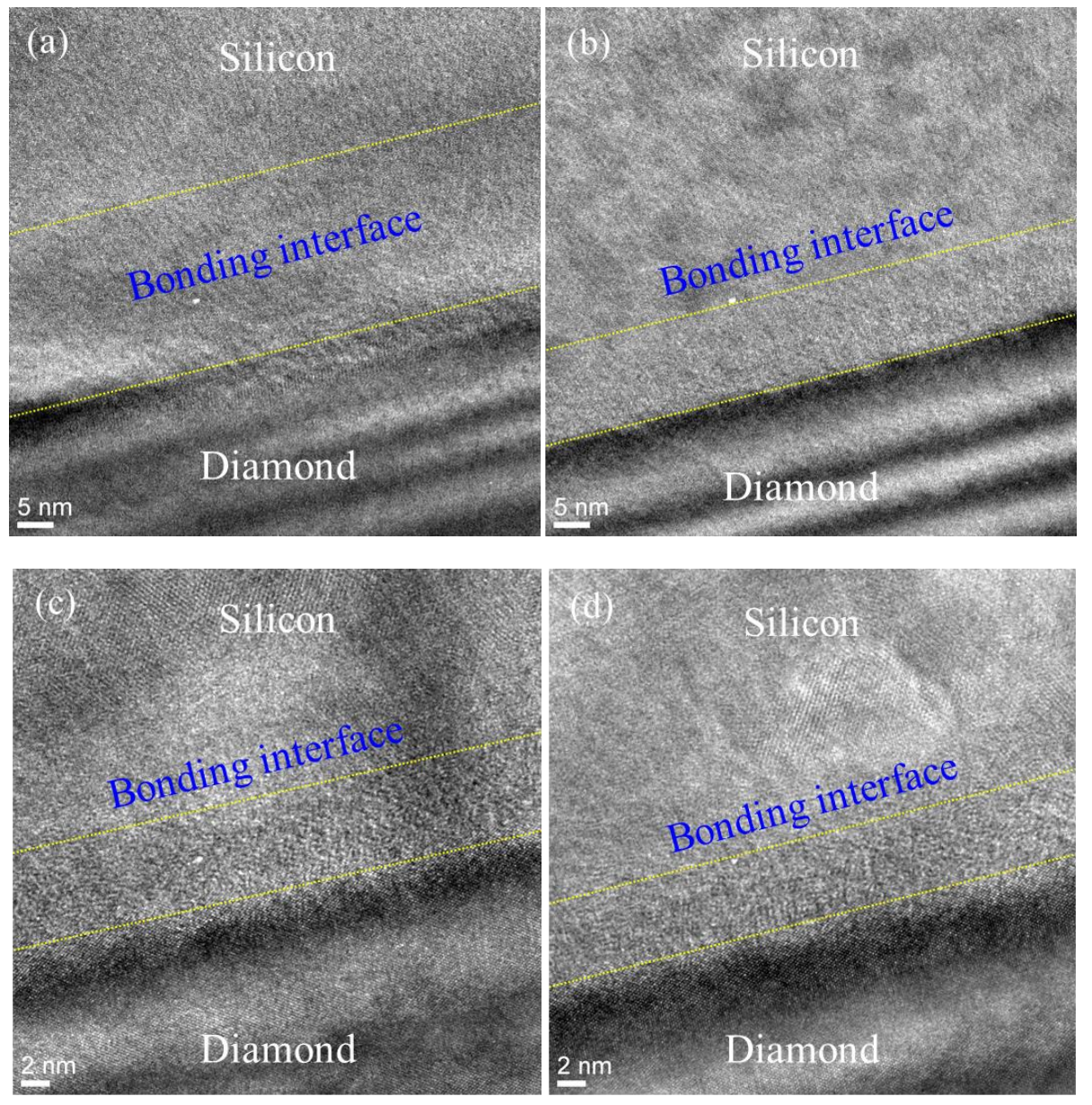

Fig. 3. High magnification cross-sectional TEM images of the diamond/Si bonding interface (a) without and with annealing at (b) 400, (c) 600, and (d) $800{ }^{\circ} \mathrm{C}$.

Raman peak shift mappings of diamond near the diamond/Si bonding interface before and after annealing at 400, 800, and $1000{ }^{\circ} \mathrm{C}$ are shown in Figs 4(a), 4(b), 4(c), and 4(d), respectively. As shown in Fig. 4(a), the Raman peak shift of diamond was found to range from 1331.78 to $1331.90 \mathrm{~cm}^{-1}$. We adopted a measured value of diamond substrate without polishing as a stress-free diamond, in which the Raman peak shift of diamond was observed at $1332.20 \mathrm{~cm}^{-1}$. Diamond of the bonding interface without annealing exhibited peak shifts to lower wave numbers with respect to that of the stress-free diamond. In addition, the Raman peak of diamond further shifted to lower wave numbers as the annealing temperature increased to $400{ }^{\circ} \mathrm{C}$. However, as the annealing temperature increased from 400 to $1000{ }^{\circ} \mathrm{C}$, the Raman peak of diamond shifted to higher wave numbers, which indicates that the residual stress in the diamond of the bonding interface released with the annealing temperature. 
(a)

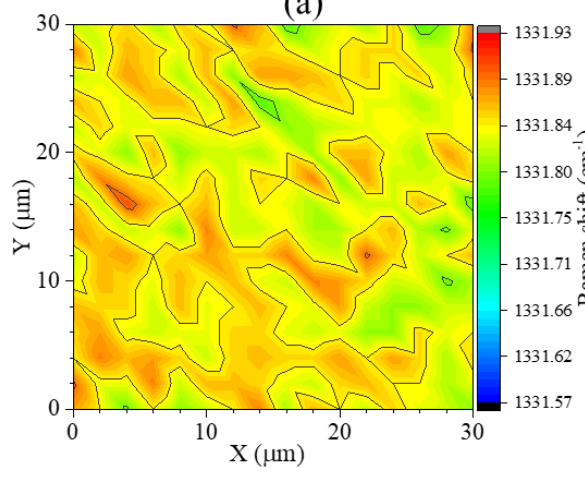

(c)

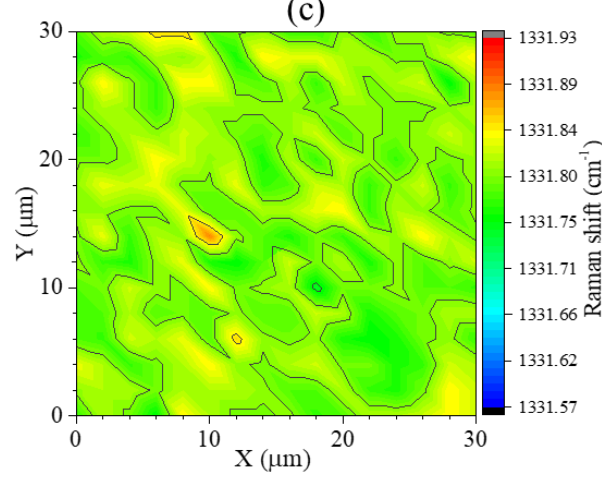

(b)

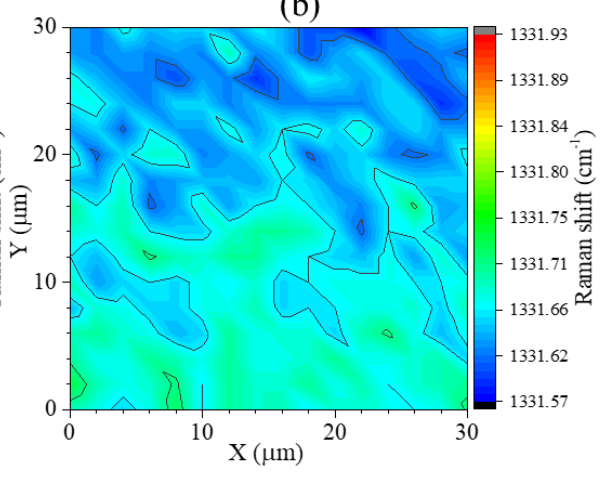

(d)

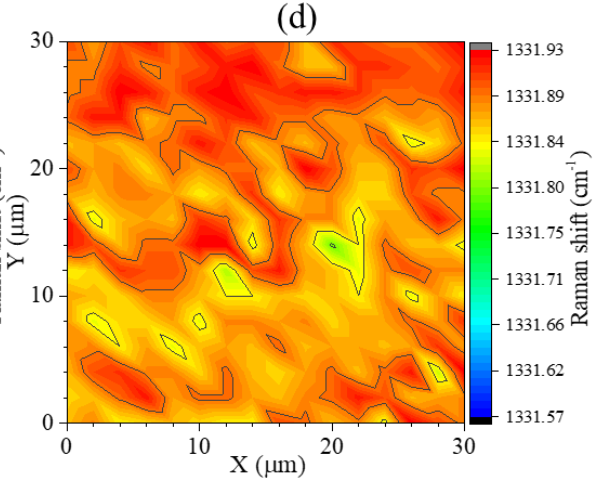

Fig. 4. Raman peak shift mappings of diamond in the bonding interface (a) without and with annealing at (b) 400 , (c) 800 and (d) $1000^{\circ} \mathrm{C}$.

The phonon frequencies of diamond and Si both shift linearly with the residual stress with 1.8 and $2.3 \mathrm{~cm}^{-1} \mathrm{GPa}^{-1}$, respectively $[26,27]$. Using these values, we calculated the magnitude of the residual stress in the diamond and $\mathrm{Si}$ of the bonding interface with respect to that of the diamond without polishing and Si before bonding, respectively. The Raman peak shift and the corresponding residual stress in the diamond and $\mathrm{Si}$ of the bonding interface with annealing at different temperatures are shown in Figs 5(a) and 5(b), respectively. The Raman peak shift and the obtained value of residual stress shown in the figures represent the averaged value over the area of $30 \times 30 \mu \mathrm{m}^{2}$. The Raman peak shift of Si was referred to the value of the $\mathrm{Si}(100)$ substrate before bonding whose peak was measured at $520.30 \mathrm{~cm}^{-1}$. The Raman peak shifts of diamond without and with polishing are also shown in the figure. 
(a)

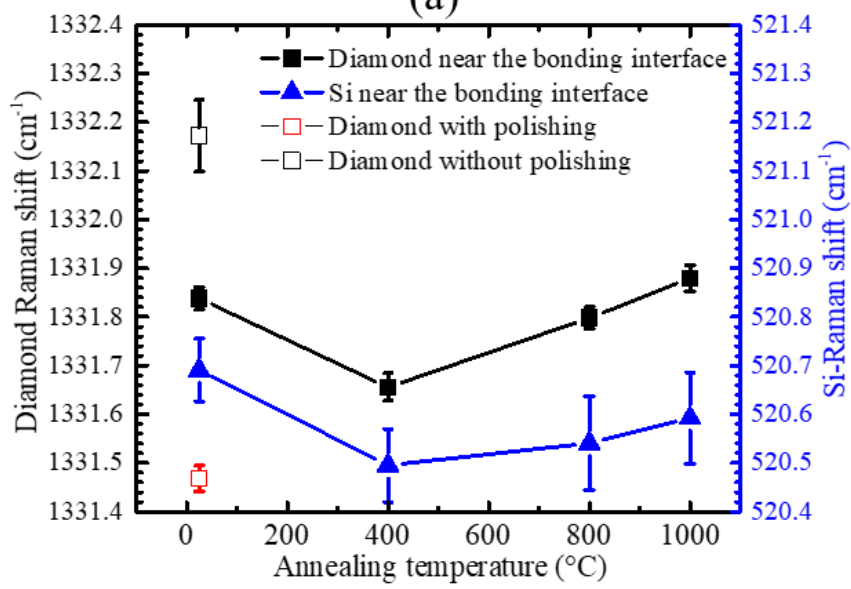

(b)

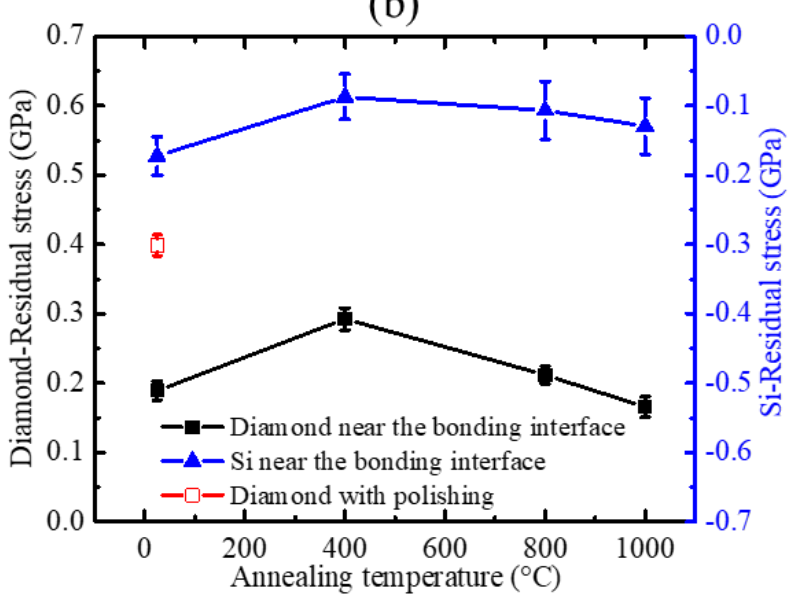

Fig. 5. (a) Raman peak shifts and (b) corresponding residual stresses of diamond and Si in the bonding interface with annealing at different temperatures.

It was found that the Raman peaks of the diamond with polishing and the diamond of the bonding interface without annealing exhibited peak shift to lower wave numbers with respect to that of the diamond without polishing, revealing that the residual stress was a tensile stress on the polished diamond surface and in the diamond of the bonding interface. The tensile stress of the polished diamond surface is much larger than that of the diamond of the bonding interface. On the other hand, the Raman peak of the Si of the bonding interface without annealing was found to shift to higher wave numbers with respect to that of the Si substrate before bonding, indicating that the residual stress in the $\mathrm{Si}$ of the bonding interface was a compressive stress. The compressive stress in the $\mathrm{Si}$ of the bonding interface decreased with increasing annealing temperature up to $400{ }^{\circ} \mathrm{C}$ and then increased as the annealing temperature increased from $400{ }^{\circ} \mathrm{C}$ to $1000{ }^{\circ} \mathrm{C}$.

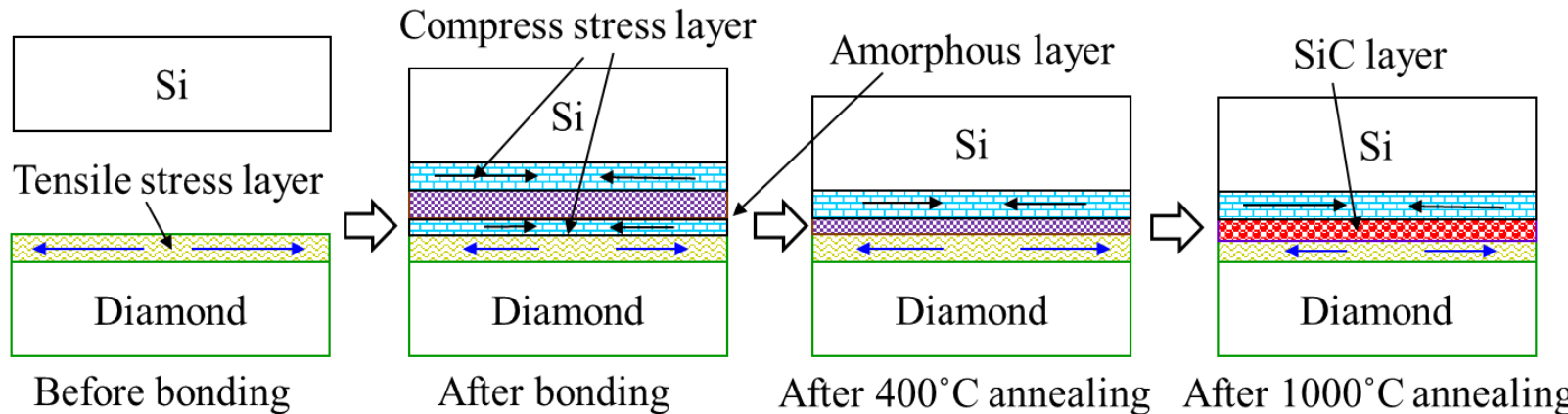

Fig. 6. Schematic illustration of residual stress change in the bonding interface without and with 
annealing at different temperatures.

One finding to emerge was that the large tensile stress observed on the polished diamond surface is normally thought to be a result of local plastic deformation induced by CMP. The tensile stress was also reported for CVD diamond wafers with polishing process [28]. Although the bonding process was achieved at room temperature, the residual stress observed in the diamond of the bonding interface without annealing is much smaller than that of the polished diamond. Furthermore, the residual stress was also observed in the Si of the bonding interface without annealing. Such results should be related to the high pressure $(8 \mathrm{GPa})$ applied to the bonding interface of diamond and Si during bonding process, which induces a compressive residual stress in diamond and $\mathrm{Si}$ of the bonding interface. The compressive stress lowered the tensile stress induced by CMP in the diamond of the bonding interface. It has been reported that the compressive stress has been generated in crystalline silicon under microindentation, the residual stress was still existed in the silicon even after removing the load [29]. Therefore, we can derive the argument that the residual stress generation and the residual stress relaxation in the $\mathrm{Si}$ and diamond of the bonding interface without annealing should be related to the applied load.

The obtained results reveal that the residual stress in the diamond and Si of the bonding interface varies with annealing temperature. The compressive stress was released in the Si of the bonding interface, on the contrary, the tensile stress was increased in the diamond of the bonding interface after annealing at $400{ }^{\circ} \mathrm{C}$. Such discrepancy is likely to be due to the compressive stress induced by the pressure was relaxed in the diamond of the bonding interface. Because the residual stresses existing in the diamond of the bonding interface were consisted of the compressive stress by the pressure and the tensile stress by CMP process. After annealing at $400{ }^{\circ} \mathrm{C}$, the compressive stress was released in the diamond and Si of the bonding interface, so that the tensile stress was recovered in the diamond. While the residual stress induced by CMP is difficult to be relaxed by annealing at temperature as low as $400{ }^{\circ} \mathrm{C}[30]$. Schematic illustration of residual stress change in the bonding interface without and with 
annealing at different temperatures is shown in Fig. 6.

The amorphous layer with a thickness of about 25 was formed at the bonding interface without annealing [Fig. 3(a)]. The amorphous layer was also reported for the Si/GaAs, GaP/GaAs, Al/diamond, and $\mathrm{Si} / \mathrm{Si}$ bonding interface fabricated by SAB [31-34]. We found that the amorphous layer thickness decreased with increasing annealing temperature. According to our previous report, the amorphous layer disappeared, and a SiC intermediate layer was formed at the diamond/Si bonding interface after annealing at $1000{ }^{\circ} \mathrm{C}[20]$. The compressive stress was increased in the $\mathrm{Si}$ of the bonding interface with the annealing temperature higher than $800{ }^{\circ} \mathrm{C}$, which should be related to the structure change of the amorphous layer formed at the bonding interface. On the other hand, the residual stress relaxation in the diamond of the bonding interface should be mainly due to the recovery of the plastic deformation layer induced by CMP. Although the thermal expansion coefficient of diamond is much smaller than that of $\mathrm{Si}$, no any structural defects such as cracks were observed at the bonding interface with annealing at different temperatures. Furthermore, the residual stress existing in the diamond and Si of the bonding interface with annealing at $1000{ }^{\circ} \mathrm{C}$ is much small in comparison with those of diamond films deposited on $\mathrm{Si}[35,36]$. These results should be attributed to the $\mathrm{SiC}$ layer formed at the bonding interface, which served as a pivotal role in the relaxation process of residual stress.

\section{Conclusion}

The effect of thermal annealing process on the interfacial structure and the residual stress of the diamond/Si bonding interface fabricated by SAB was investigated by In-situ TEM observation and micro-Raman Spectroscopy, respectively. It was found that an amorphous layer with a thickness of about $25 \mathrm{~nm}$ was formed at the bonding interface, the amorphous layer thickness decreased as increasing annealing temperature, which decreased to $5.6 \mathrm{~nm}$ after annealing at $800{ }^{\circ} \mathrm{C}$. No cracks and voids in the atomic scale were observed at the bonding interface with annealing at various temperatures. The residual stress in the $\mathrm{Si}$ of the bonding interface without annealing increased, while the residual 
stress in the diamond of the bonding interface without annealing decreased with respect to those of the bonding Si and the polished diamond, respectively. These results were attributed to the load applied to the bonding substrates during bonding process. After annealing at $1000{ }^{\circ} \mathrm{C}$, the residual stress existing in the bonding interface was very small to take the thermal expansion coefficient difference between diamond and $\mathrm{Si}$ into account, which was ascribed to the $\mathrm{SiC}$ layer formed at the bonding interface.

\section{Acknowledgements}

This study was supported partly by Hirose International Scholarship Foundation and the Grant-inAid for Challenging Exploratory Research (16K13676) of the Ministry of Education, Culture, Sports, Science, and Technology (MEXT), Japan. Bristol acknowledges partial financial support from the EPSRC Programme Grant GaN-DaME (EP/P00945X/1).

\section{References}

[1] J. Isberg, J. Hammersberg, E. Johanssion, T. Wikström, D. J. Twitchen, A. J. Whitehead, S. E. Core, and G. A. Scarsbrook, High Carrier Mobility in Single-Crystal Plasma-Deposited Diamond, Science 297 (2002) 1670-1672.

[2] A. T. Collins, In Properties and Growth of Diamond, IEE: Inspec, London, 1994.

[3] H. Umezawa, Y. Kato, and S. I. Shikata, $1 \Omega$ on-resistance diamond vertical-Schottky barrier diode Schottky barrier diode operated at $250{ }^{\circ} \mathrm{C}$, Appl. Phys. Exp. 6 (2013) 011302.

[4] T. Makino, S. Tanimoto, Y. Hayashi, H. Kato, N. Tokuda, M. Ogura, D. Takeuchi, K. Oyama, H. Ohashi, H. Okushi, and S. Yamasaki, Diamond Schottky-pn diode with high forward current density and fast switching operation, Appl. Phys. Lett. 94 (2009) 262101.

[5] W. Ebert, A. Vescan, T. H. Borst, E. Kohn, High current p/p/sup +/-diamond Schottky diode, IEEE Electron Device Lett. 15 (1994) 289-291. 
[6] K. Ikeda, H. Umezawa, K. Ramanujam, and S. I. Shikata, Thermally Stable Schottky Barrier Diode by Ru/Diamond, App. Phys. Express 2 (2009) 011202.

[7] M. Kasu, H. Sato, and K. Hirama, Thermal Stabilization of Hole Channel on H-Terminated Diamond Surface by Using Atomic-Layer-Deposited $\mathrm{Al}_{2} \mathrm{O}_{3}$ Overlayer and its Electric Properties, App. Phys. Express 5 (2012) 025701.

[8] T. Funaki, M. Hirano, H. Umezawa, and S. Shikata, High temperature switching operation of a power diamond Schottky barrier diode, IEICE Electron Express 9 (2012) 1835-1841.

[9] C. S. Yan, Y. K. Vohra, H. K. Mao, R. J. Hemley, Very high growth rate chemical vapor deposition of single-crystal diamond, Proc. Nat. Acad. Sci. 99 (2002) 12523-12525.

[10] Y. Mokuno, A. Chayahara, H. Yamada, N. Tsubouchi, Improving purity and size of single-crystal diamond plates produced by high-rate CVD growth and lift-off process using ion implantation, Diamond Relat. Mater. 18 (2009) 1258-1261.

[11] Q. Liang, C. S. Yan, Y. Meng. J. Lai, S. Krasnicki, H. K. Mao, R. J. Hemley, Recent advances in high-growth rate single-crystal CVD diamond, Diamond Relat. Mater. 18 (2009) 698-703.

[12] M. Mayr, C. Stehl, M. Fischer, S. Gsell, and M. Schreck, Correlation between surface morphology and defect structure of heteroepitaxial diamond grown on off-axis substrates, Phys. Status Solidi A, $211(2014) 2257$.

[13] O. Klein, M. Mayer, M. Fischer, S. Gsell, and M. Schreck, Propagation and annihilation of threading dislocations during off-axis growth of heteroepitaxial diamond films, Diamond Relat. Mater. 65 (2016) 53.

[14] T. Suto, J. Yaita, T. Iwasaki, and M. Hatano, Highly oriented diamond (111) films synthesized by pulse bias-enhanced nucleation and epitaxial grain selection on a 3C-SiC/Si (111) substrate, Appl. Phys. Lett. 110 (2017) 062102.

[15] A. Samoto, S. Ito, A. Hotta, T. Hasebe, Y. Ando, A. Sawabe, and T. Suzuki, Investigation of heterostructure between diamond and iridium on sapphire, Diamond Relat. Mater. 17 (2008) 1039- 
1044.

[16] S. Koizumi, C. Nebel, and M. Nesladek, Physics and Application of CVD Diamond, Wiley-VCH, Weinheim, 2008.

[17] M. Schreck, Comprehensive Hard Materials, In V. K. Sarin \& C. E. Nebel, Single Crystal Diamond Growth on Iridium, Elsevier, 2014, pp. 269-304.

[18] B. -C. Gallheber, M. Fischer, M. Mayr, J. Straub, and M. Schreck, Growth, stress, and defects of heteroepitaxial diamond on Ir/YSZ/Si(111), J. Appl. Phys. 123 (2018) 225302.

[19] J. Liang, S. Masuya, M. Kasu, and N. Shigekawa, Realization of direct bonding of single crystal diamond and Si substrates, Appl. Phys. Lett. 110 (2017) 111603.

[20] J. Liang, S. Masuya, S. Kim, T. Oishi, M. Kasu, and N. Shigekawa, Stability of diamond/Si bonding interface during device fabrication process, Appl. Phys. Express 12 (2019) 016501.

[21] M. M. R. Howlader and F. Zhang, Void-free strong bonding of surface activated silicon wafers from room temperature to annealing at $600{ }^{\circ} \mathrm{C}$, Thin Solid Films 519 (2010) 804-808.

[22] J. Liang, S. Nishida, M. Arai, and N. Shigekawa, Effects of thermal annealing process on the electrical properties of $\mathrm{p}^{+}-\mathrm{Si} / \mathrm{n}-\mathrm{SiC}$ heterojunctions, Appl. Phys. Lett. 104 (2014) 161604.

[23] H. Takagi, R. Maeda, N. Hosoda, and T. Suga, Transmission Electron Microscope Observations of Si/Si Interface Bonded at Room Temperature by Ar Beam Surface Activation, Jpn. J. Appl. Phys. 38 (1999) 1589.

[24] J. Liang, T. Miyazaki, M. Morimoto, S. Nishida, N. Watanabe, and N. Shigekawa, Electrical Properties of $\mathrm{p}-\mathrm{Si} / \mathrm{n}-\mathrm{GaAs}$ Heterojunctions by Using Surface-Activated Bonding, Appl. Phys. Express $6(2013) 021801$.

[25] H. Takagi, K. Kikuchi, R. Maeda, T. R. Chung, and T. Suga, Surface activated bonding of silicon wafers at room temperature, Appl. Phys. Lett. 68 (1996) 2222.

[26] J. W. Ager III and M. D. Drory, Quantitative measurement of residual biaxial stress by Raman spectroscopy in diamond grown on a Ti alloy by chemical vapor deposition, Phys. Rev. B 48 (1993) 
2601.

[27] J. Chen and I. D. Wolf, Study of damage and stress induced by backgrinding in Si wafers, Semicond. Sci. Technol. 18 (2003) 261.

[28] Y. Chen, W. Liu, H. Feng, and L. Zhang, Failure Mechanisms of CVD Diamond Wafers and Thin Films During Polishing, Machining Science and Technology 19 (2015) 152-173.

[29] G. Lucazeau and L. Abello, Micro-Raman analysis of residual stresses and phase transformations in crystalline silicon under microindentation, J. Mater. Res. 12 (1997) 2262-2273.

[30] X. Chen, X. Xu, X. Xu, J. Li, Y. Wang, S. Jiang, K. Zhang, and M. Jiang, Relaxation of residual stresses in $\mathrm{SiC}$ wafers by annealing, Rare Metals, 25 (2006) 704-708.

[31] J. Liang, L. Chai, S. Nishida, M. Morimoto, and N. Shigekawa, Investigation on the interface resistance of $\mathrm{Si} / \mathrm{GaAs}$ heterojunctions fabricated by surface-activated bonding, Jpn. J. Appl. Phys. 54 (2015) 030211.

[32] M. M. R. Howlader, T. Suga, F. Zhang, T. H. Lee, and M. J. Kim, Interfacial Behavior of Surface Activated p-GaP/n-GaAs Bonded Wafers at Room Temperature, Electrochem. Solid-State Lett. 13 (2010) H61-H65.

[33] J. Liang, S. Yamajo, M. Kuball, N. Shigekawa, Room-temperature direct bonding of diamond and Al, Scripta Materialia 159 (2019) 58-61.

[34] J. Liang, T. Miyazaki, M. Morimoto, S. Nishida, and N. Shigekawa, Electrical properties of Si/Si interfaces by using surface-activated bonding, J. Appl. Phys. 114 (2013) 183703.

[35] T. Guillemet, Z. Q. Xie, Y. S. Zhou, J. B. Park, A. Veillere, W. Xiong, J. M. Heintz, J. F. Silvain, N. Chandra, and Y. F. Lu, Stress and Phase Purity Analyses of Diamond Films Deposited through Laser-Assisted Combustion Synthesis, ACS Appl. Mater. Interfaces 3 (2011) 4120-4125.

[36] N. G. Ferreira, E. Abramof, N. F. Leite, E. J. Corat, and V. J. Trava-Airoldi, Analysis of residual stress in diamond films by x-ray diffraction and micro-Raman spectroscopy, J. Appl. Phys. 91 (2002) $2466(2002)$. 